\title{
CT-PPS Physics Results and Prospects
}

\author{
Justin Williams* \\ On behalf of the CMS and TOTEM Collaborations \\ E-mail: justinwilliams@ku.edu
}

\begin{abstract}
A unique search for physics beyond the standard model in exclusive diphoton events is presented. By measuring intact protons from the events in the forward region, we can significantly reduce the background, leading to the best sensitivity to anomalous coupling behavior. Furthermore, by studying the process of $\gamma \gamma \rightarrow \gamma \gamma$ we can shed light on the high mass regions of Axion-like particles, and potential dark matter candidates.
\end{abstract}

2nd World Summit: Exploring the Dark Side of the Universe

25-29 June, 2018

University of Antilles, Pointe-á-Pitre, Guadeloupe, France

${ }^{*}$ Speaker. 


\section{Introduction}

Typically the LHC is used as a proton-proton collider; however, there is such a large amount of photons produced in the beam pipe that we can use the LHC as a photon-photon collider. Events in which photons collide are interesting to study because they may be sensitive to beyond standard model (BSM) physics[1][2]. We propose to use the CMS Precision Proton Spectrometer (CT-PPS) to study these exciting events in a unique way by using proton tagging techniques. The tagging of outgoing protons allows for a strong background suppresion that we will see leads to a great sensitivity in the high mass region of diphoton events.

The paper begins with a description of CT-PPS followed by an overview of the first physics results. Then there will be a discusion of the backgrounds and motivations before the conclusion.

\section{CMS Precision Proton Spectrometer}

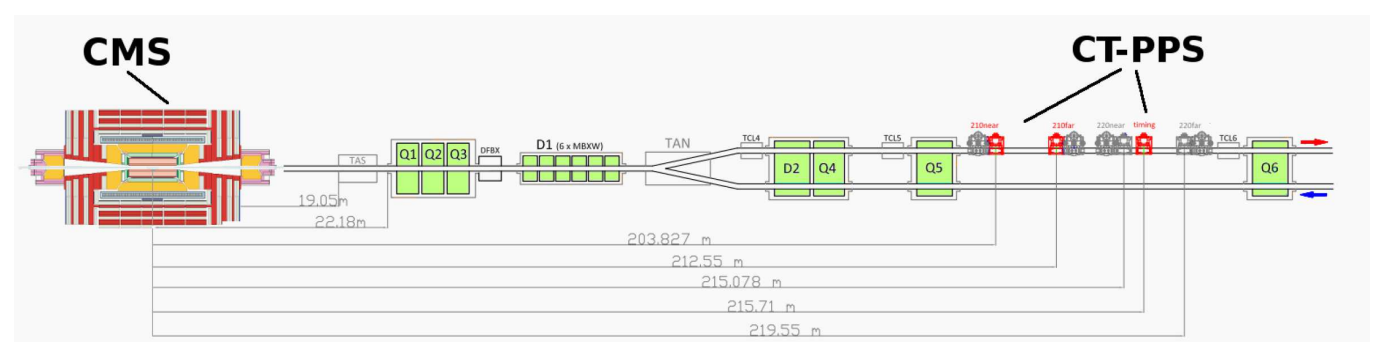

Figure 1: A schematic of one side of CT-PPS. Immediately to the right of the CMS detector are the LHC magnets. Further to the right are the Roman Pot stations where intact protons are detected.

Originally a joint project between CMS and TOTEM[3], CT-PPS is designed to measure intact protons on either side of CMS, about $200 \mathrm{~m}$ away from the interaction point. After an interaction in CMS, protons are pushed just outside of the beam envelope by the LHC magnets due to the loss of momentum. These protons are then detected further downstream where the momentum loss can be measured based on the position where the protons are found. The specific variable of interest to be calculated is the fractional momentum loss $\xi$ which can be used to correlate the forward protons with the central system within CMS.

The detectors are housed within Roman Pots, which use a combination of silicon strips and pixels to measure the $\mathrm{x}$ and $\mathrm{y}$ positions of the protons. Fig. 1 shows one side of the CT-PPS system. A symmetric set of detectors is present on the other side as well.

\section{Physics Results From CT-PPS}

The first analysis published using CT-PPS was a study of photon-induced dilepton events. This was the first observation of this proccess at high mass using intact protons. Fig. 2 shows three different processes generating opposite sign leptons by way of photon-photon interactions. For the sake of this analysis, the first two processes are treated as signal whereas the third case where both protons dissociate, was treated as a background. This analysis was performed at nominal optics and pileup conditions for the LHC and yielded results that were recently published[4]. 

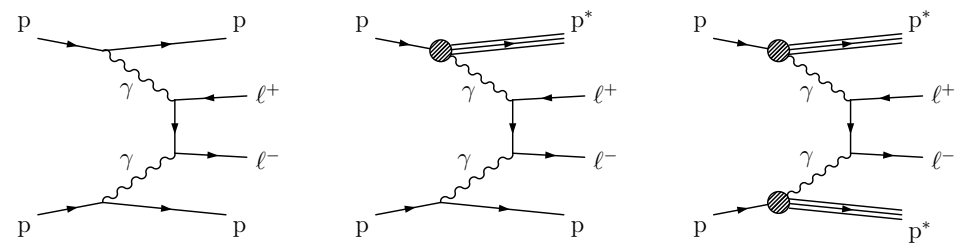

Figure 2: Feyman diagrams of events producing two opposite sign leptons in the final state. For the process on the far left, both protons remain intact in the final state. For the middle process, only one proton remains intact while the other proton dissociates. On the right is the case when both protons dissociate.
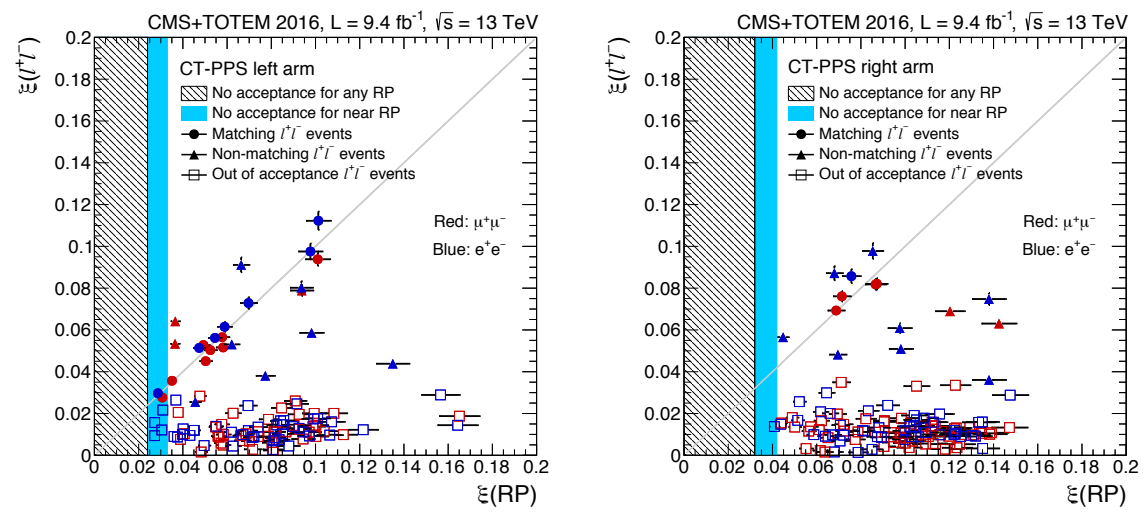

Figure 3: Both plots show dilepton events that were found with at least one corresponding proton measured in CT-PPS. The left plot is for events with a proton detected on the left side of CMS and the right plot is for events with a proton detected on the right side of CMS. The diagonal line is where events will be that have a perfect matching between the $\xi$ value of the central system and the $\xi$ value of the forward proton(s)[4].

The events that were observed can be seen along the diagonal line on the plots in Fig. 3. Events along the diagonal line are events which have a matching between the $\xi$ value of the central leptons and the $\xi$ measurement of the forward protons in CT-PPS. $12 \mu^{+} \mu^{-}$events and $8 \mathrm{e}^{+} \mathrm{e}^{-}$ events were observed. Compared with the expected background of $1.49 \pm 0.07$ (stat) \pm 0.53 (syst) for $\mu^{+} \mu^{-}$events and $2.36 \pm 0.09$ (stat) \pm 0.47 (syst) for $\mathrm{e}^{+} \mathrm{e}^{-}$events, this is an observation at the $5.1 \sigma$ level.

The success of this analysis indicates that the alignment, triggering, and optics are all well understood within CT-PPS and that the concept of forward proton tagging can be very succesful for other analyses.

\section{Anomalous Quartic Gauge Couplings}

With the proton tagging techniques in CT-PPS comes the oppurtunity to search for anomalous quartic gauge couplings (AQGC) in a unique way. Fig. 4 shows three such couplings that can be explored using proton tagging. The four-photon anomalous coupling will be the main focus of this discussion as it is sensitive to other BSM couplings that will be discussed in section 6 . The characteristics of these events that we can explot for this analysis are the intact protons in the final state, and the exclusive diboson production within CMS. The power of this study is that we can analyse the totality of the process by measuring every final state particle. 

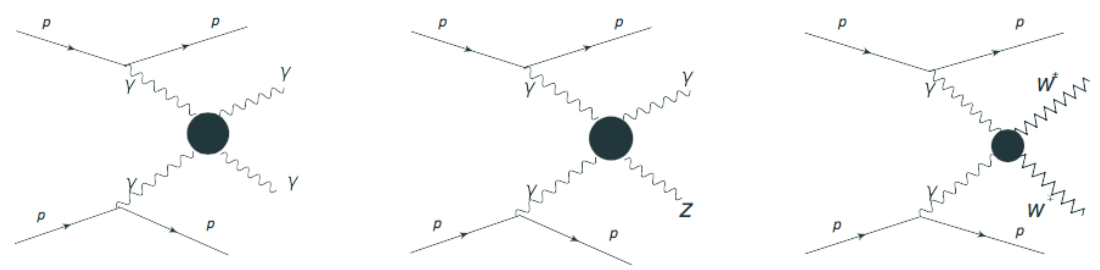

Figure 4: Feyman diagrams of three different anomalous quartic gauge couplings resulting from photon fusion. From left to right, the diagrams are $\gamma \gamma \rightarrow \gamma \gamma, \gamma \gamma \rightarrow \gamma \mathrm{Z}, \gamma \gamma \rightarrow \mathrm{W}^{+} \mathrm{W}^{-}$.

\section{Backgrounds}

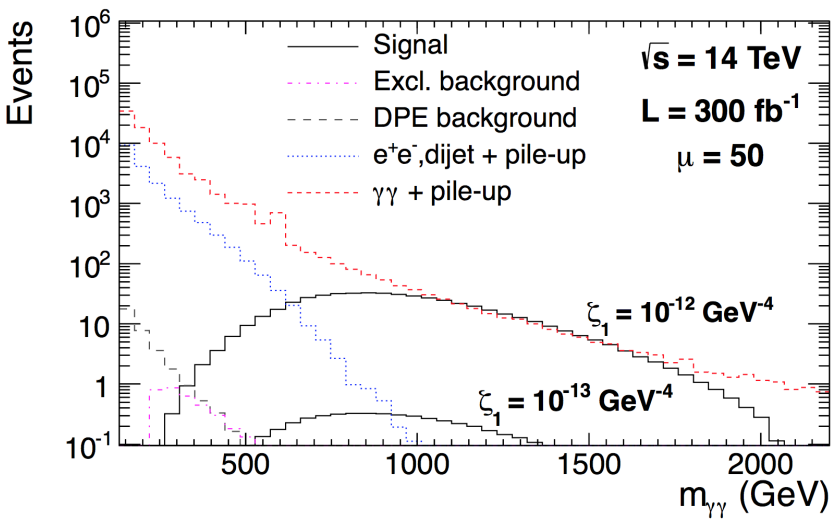

Figure 5: A histogram for various types of backgrounds (colored lines) with respect to two signals of different coupling values. The histogram is plotted as a function of the diphoton mass[6].

The signal for the process of interest consists of two protons identified in the forward detectors in coincidence with two photons in the central detector. Using the Forward Physics Monte Carlo (FPMC)[5], Fig 5 shows the significance of the types of backgrounds that could fake the signal[6]. While all backgrounds are considered such as double pomeron exchange, $\mathrm{H} \rightarrow \gamma \gamma$, misidentification of leptons and jets, pileup, Drell-Yan, etc., the only background that makes a significant contribution at high diphoton mass is that from pileup.

At the LHC, packets of protons are collided per bunch crossing. While our intent is to detect protons coming from the same event as the process that created two photons, sometimes this is not the case. For the case of the pileup background, two photons may be created in CMS, but protons may be measured that actually come from an unrelated event. This overlapping of uncorrelated events creates a very significant background: for example, in 2016, there were up to 50 pileup vertices per bunch crossing.

The way we can suppress this pileup background is by exploiting momentum conservation. Specifically, the mass and rapidity of the centrally produced system as measured in the central CMS apparatus and in CT-PPS are required to match. Fig. 6 shows that by requiring this matching, the signal peaks well above the background coming from pileup. 

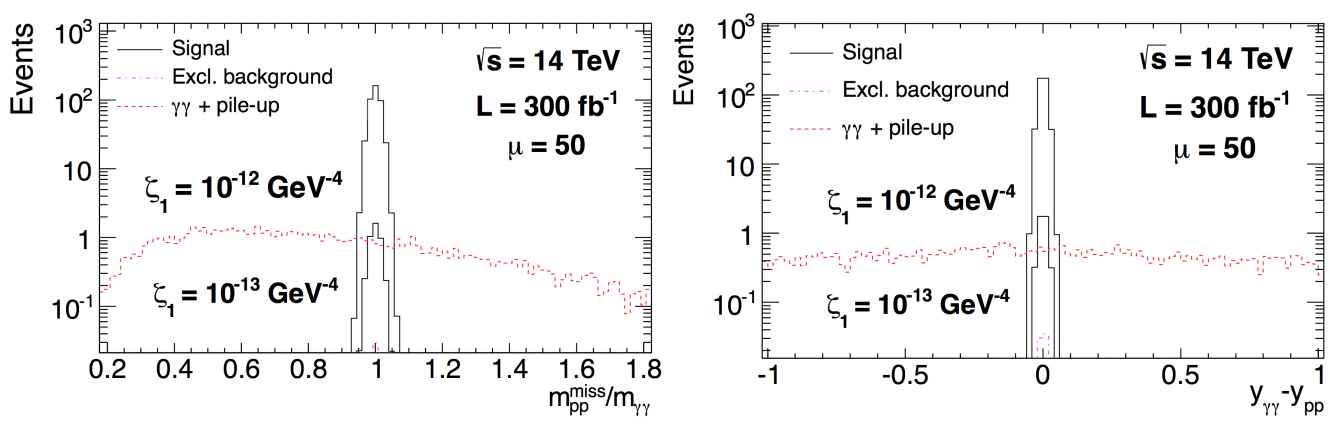

Figure 6: Diphoton to missing proton mass ratio (left) and rapidity difference (right) distributions for signal considering two different coupling values $\left(10^{-12}\right.$ and $\left.10^{13} \mathrm{GeV}^{-4}\right)$ and for backgrounds after requirements on photon pT, diphoton invariant mass, pT ratio between the two photons and on the angle between the two photons. The integrated luminosity is $300 \mathrm{fb}^{-1}$ and the average pileup is $\mu=50[6]$.

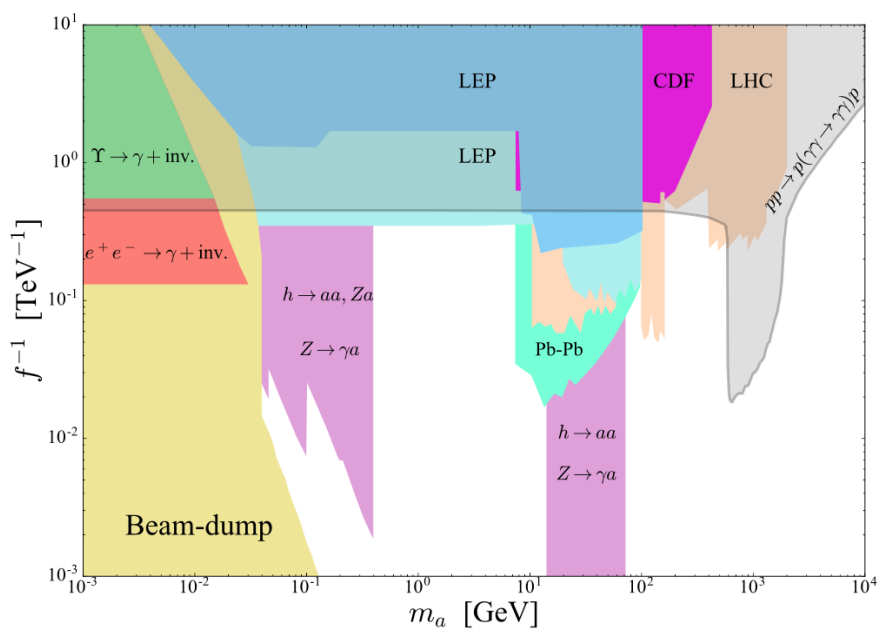

Figure 7: A plot of the current exclusion regions for ALPs from various different experiments. On the horizontal axis is the ALP mass and on the vertical axis is the coupling strength[7].

\section{Applications}

An additional benefit of studying four-photon anomalous couplings is the extra insight on BSM models of axion-like particles (ALP) and dark matter candidates. We can explore new mass regimes of ALP production via photon exchange using proton tagging in CT-PPS[7]. This search even improves upon the standard mass reach of the LHC for ALPs. Fig. 7 shows the kinematic regimes that have already been excluded from various experiments such as astroparticle and collider searches. The figure also shows the region that can be explored by means of proton tagging.

In a similar way, we can study the case in which a dark matter particle - a polarizable dark particle - is the mediator in the four-photon loop. It is proposed to use the $\gamma \gamma \gamma \gamma$ coupling to search for such a particle as it automatically induces four photon legs[8]. It has been shown that there is potential to discover the existence of this particle at a $5 \sigma$ significance at the LHC[8]. 


\section{Conclusion}

In 2016, CT-PPS showed for the first time the feasability of operating a near-beam proton spectrometer at a high-luminosity collider on a regular basis. From the data that has been acquired, the first observation was made of the process $\gamma \gamma \rightarrow l^{+} l^{-}$using proton tagging. The success of the observation has paved the way for BSM searches of anomalous couplings, ALPs, dark matter, and other interesting physics. Through 2016 and 2017, CT-PPS has taken over $55 \mathrm{fb}^{-1}$ of data and has plans to continue this level of progress going forward.

\section{References}

[1] D. d'Enterria and G. G. da Silveira, Phys. Rev. Lett. 111, 080405 (2013) Erratum: [Phys. Rev. Lett. 116, no. 12, 129901 (2016)] doi:10.1103/PhysRevLett.111.080405, 10.1103/PhysRevLett.116.129901 [arXiv:1305.7142 [hep-ph]].

[2] J. de Favereau de Jeneret et al., arXiv:0908.2020 [hep-ph].

[3] M. Gallinaro [CMS and TOTEM Collaborations], AIP Conf. Proc. 1819, no. 1, 040021 (2017) doi:10.1063/1.4977151 [arXiv:1611.07431 [physics.ins-det]].

[4] A. M. Sirunyan et al. [CMS and TOTEM Collaborations], JHEP 1807, 153 (2018) doi:10.1007/JHEP07(2018)153 [arXiv:1803.04496 [hep-ex]].

[5] M. Boonekamp, A. Dechambre, V. Juranek, O. Kepka, M. Rangel, C. Royon and R. Staszewski, arXiv:1102.2531 [hep-ph].

[6] S. Fichet, G. von Gersdorff, O. Kepka, B. Lenzi, C. Royon and M. Saimpert, Phys. Rev. D 89, 114004 (2014) doi:10.1103/PhysRevD.89.114004 [arXiv:1312.5153 [hep-ph]].

[7] C. Baldenegro, S. Fichet, G. von Gersdorff and C. Royon, JHEP 1806, 131 (2018) doi:10.1007/JHEP06(2018)131 [arXiv:1803.10835 [hep-ph]].

[8] S. Fichet, JHEP 1704, 088 (2017) doi:10.1007/JHEP04(2017)088 [arXiv:1609.01762 [hep-ph]]. 